\title{
On Weakly Singular Versions of Discrete Nonlinear Inequalities and Applications
}

\author{
Kelong Cheng, ${ }^{1}$ Chunxiang Guo, ${ }^{2}$ and Qingke Zeng' \\ ${ }^{1}$ School of Science, Southwest University of Science and Technology, Mianyang 621010, China \\ ${ }^{2}$ School of Business, Sichuan University, Chengdu 610064, China \\ Correspondence should be addressed to Chunxiang Guo; guocx70@163.com
}

Received 17 March 2014; Accepted 13 August 2014; Published 27 August 2014

Academic Editor: Jehad Alzabut

Copyright (c) 2014 Kelong Cheng et al. This is an open access article distributed under the Creative Commons Attribution License, which permits unrestricted use, distribution, and reproduction in any medium, provided the original work is properly cited.

Some new weakly singular versions of discrete nonlinear inequalities are established, which generalize some existing weakly singular inequalities and can be used in the analysis of nonlinear Volterra type difference equations with weakly singular kernels. A few applications to the upper bound and the uniqueness of solutions of nonlinear difference equations are also involved.

\section{Introduction}

Recently, along with the development of the theory of integral inequalities and difference equations, many authors have researched some discrete versions of Gronwall-Bellman type inequalities [1-5]. Starting from the basic form,

$$
u(n) \leq a(n)+\sum_{s=0}^{n-1} f(s) u(s)
$$

discussed originally by Pachpatte in [4], various such new inequalities have been established, which can be used as a powerful tool in the analysis of certain classes of finite difference equations. Among these results, discrete weakly singular integral inequalities also play an important role in the study of the behavior and numerical solutions for singular integral equations $[6,7]$ and the theory for parabolic equations [8-10]. For example, Dixon and McKee [7] investigated the convergence of discretization methods for the Volterra integral and integrodifferential equations using the following inequality:

$$
\begin{array}{r}
x_{i} \leq \psi_{i}+M h^{1-\alpha} \sum_{j=0}^{i-1} \frac{x_{j}}{(i-j)^{\alpha}}, \quad i=1,2, \ldots, N, \\
n>0, \quad N h=T,
\end{array}
$$

and Beesack [6] also discussed the inequality,

$$
x_{i} \leq \psi_{i}+M h^{1+\sigma-\alpha \beta} \sum_{j=0}^{i-1} \frac{j^{\sigma} x_{j}}{\left(i^{\beta}-j^{\beta}\right)^{\alpha}},
$$

for the second kind Abel-Volterra singular integral equations. Henry [9] presented a linear inequality

$$
x_{n} \leq a_{n}+\sum_{k=0}^{n-1}\left(t_{n}-t_{k}\right)^{\beta-1} \tau_{k} b_{k} x_{k},
$$

to investigate some qualitative properties for a parabolic equation. In particular, to avoid the shortcoming of analysis, Medved [11-13] used a new method to discuss some nonlinear weakly singular integral inequalities and difference inequalities. Following Medved's work, Ma and Yang [14] improved his method to discuss a more general nonlinear weakly singular integral inequality,

$$
\begin{aligned}
u(t) \leq & a(t)+b(t) \int_{0}^{t}\left(t^{\sigma}-s^{\sigma}\right)^{\mu-1} s^{\tau-1} g(s) u(s) d s \\
& +c(t) \int_{0}^{t}\left(t^{\alpha}-s^{\alpha}\right)^{\beta-1} s^{\gamma-1} g(s) w(u(s)) d s,
\end{aligned}
$$


and a nonlinear difference inequality [15],

$$
x_{n}^{\alpha} \leq a_{n}+\sum_{k=0}^{n-1}\left(t_{n}-t_{k}\right)^{\beta-1} \tau_{k} b_{k} x_{k}^{\lambda}
$$

As for other new weakly singular inequalities, recent work can be found, for example, in [16-25] and references therein.

In this paper, we investigate some new nonlinear discrete weakly singular inequalities

$$
\begin{array}{r}
x_{n} \leq a_{n}+\sum_{k=0}^{n-1}\left(t_{n}^{\alpha}-t_{k}^{\alpha}\right)^{\beta-1} t_{k}^{\gamma-1} \tau_{k} b_{k} \omega\left(x_{k}\right), \\
x_{n} \leq a_{n}+\sum_{k=0}^{n-1}\left(t_{n}^{\sigma}-t_{k}^{\sigma}\right)^{\mu-1} t_{k}^{\lambda-1} \tau_{k} g_{k} x_{k} \\
+\sum_{k=0}^{n-1}\left(t_{n}^{\alpha}-t_{k}^{\alpha}\right)^{\beta-1} t_{k}^{\gamma-1} \tau_{k} b_{k} \omega\left(x_{k}\right) .
\end{array}
$$

Compared to the existing result, our result is more concise and can be used to obtain pointwise explicit bounds on solutions of a class of more general weakly singular difference equations of Volterra type. Finally, to illustrate the usefulness of the result, we give some applications to Volterra type difference equation with weakly singular kernels.

For convenience, before giving our main results, we first cite some useful lemmas here.

Lemma 1 (discrete inequality, see [15]). Let $A_{1}, A_{2}, \ldots, A_{n}$ be nonnegative real numbers and $r>1$ a real number. Then

$$
\left(A_{1}+A_{2}+\cdots+A_{n}\right)^{r} \leq n^{r-1}\left(A_{1}^{r}+A_{2}^{r}+\cdots+A_{n}^{r}\right) .
$$

Lemma 2 (discrete Hölder inequality, see [15]). Let $a_{i}, b_{i}$ $(i=1,2, \ldots, n)$ be nonnegative real numbers, and $p, q$ positive numbers such that $1 / p+1 / q=1$ (or $p=1, q=\infty)$. Then

$$
\sum_{i=1}^{n} a_{i} b_{i} \leq\left(\sum_{i=1}^{n} a_{i}^{p}\right)^{1 / p}\left(\sum_{i=1}^{n} b_{i}^{q}\right)^{1 / q} .
$$

Lemma 3 (see [14]). Let $\alpha, \beta, \gamma$, and $p$ be positive constants. Then

$$
\begin{aligned}
& \int_{0}^{t}\left(t^{\alpha}-s^{\alpha}\right)^{p(\beta-1)} s^{p(\gamma-1)} d s \\
& \quad=\frac{t^{\theta}}{\alpha} B\left[\frac{p(\gamma-1)+1}{\alpha}, p(\beta-1)+1\right], \quad t \in R_{+},
\end{aligned}
$$

where $B[\xi, \eta]=\int_{0}^{1} s^{\xi-1}(1-s)^{\eta-1} d s(\operatorname{Re} \xi>0, \operatorname{Re} \eta>0)$ is the well-known B-function and $\theta=p[\alpha(\beta-1)+\gamma-1]+1$.

In what follows, denote $\mathbb{R}$ to be the set of real numbers. Let $\mathbb{R}_{+}=(0, \infty)$ and $\mathbb{N}=\{0,1,2, \ldots\}$. $C(X, Y)$ denotes the collection of continuous functions from the set $X$ to the set $Y$. As usual, the empty sum is taken to be 0 .

\section{Some New Nonlinear Weakly Singular Difference Inequalities}

Lemma 4. Suppose that $\omega(u) \in C\left(\mathbb{R}_{+}, \mathbb{R}_{+}\right)$is nondecreasing with $w(u)>0$ for $u>0$. Let $a_{n}, c_{n}$ be nonnegative and nondecreasing in $n$. If $y_{n}$ is nonnegative such that

$$
y_{n} \leq a_{n}+c_{n} \sum_{k=0}^{n-1} b_{k} \omega\left(y_{k}\right), \quad n \in \mathbb{N},
$$

then

$$
y_{n} \leq \Omega^{-1}\left[\Omega\left(a_{n}\right)+c_{n} \sum_{k=0}^{n-1} b_{k}\right], \quad 0 \leq n \leq M,
$$

where $\Omega(v)=\int_{v_{0}}^{v}(1 / \omega(s)) d s, v \geq v_{0}, \Omega^{-1}$ is the inverse function of $\Omega$, and $M$ is defined by

$$
M=\sup \left\{i: \Omega\left(a_{i}\right)+c_{i} \sum_{k=0}^{i-1} b_{k} \in \operatorname{Dom}\left(\Omega^{-1}\right)\right\} .
$$

Assume that

$\left(S_{1}\right) \alpha \in(0,1], \beta \in(0,1)$ and $1>(p(\gamma-1)+1) / \alpha \geq$ $p(\beta-1)+1>0$ such that $1 / p+\alpha(\beta-1)+\gamma-1 \geq 0 ;$

$\left(S_{2}\right) a_{n}, b_{n}$ are nonnegative functions for $n \in \mathbb{N}$, respectively;

$\left(S_{3}\right) \omega(u) \in C\left(\mathbb{R}_{+}, \mathbb{R}_{+}\right)$is nondecreasing and $\omega(0)=0$.

Lemma 5. Suppose that $[\alpha, \beta, \gamma]$ satisfies assumption $\left(S_{1}\right)$; then for sufficiently small $\tau_{k}$, one has

$$
\begin{aligned}
& \sum_{k=0}^{n-1}\left(t_{n}^{\alpha}-t_{k}^{\alpha}\right)^{p_{i}(\beta-1)} t_{k}^{p_{i}(\gamma-1)} \tau_{k} \\
& \quad \leq \int_{0}^{t_{n}}\left(t_{n}^{\alpha}-s^{\alpha}\right)^{p_{i}(\beta-1)} s^{p_{i}(\gamma-1)} d s \\
& \quad=\frac{t_{n}^{\theta_{i}}}{\alpha} B\left[\frac{p_{i}(\gamma-1)+1}{\alpha}, p_{i}(\beta-1)+1\right] .
\end{aligned}
$$

Proof. Consider the B-function in (15). Consider

$$
\begin{gathered}
B\left[\frac{p(\gamma-1)+1}{\alpha}, p(\beta-1)+1\right] \\
\quad=\int_{0}^{1}(1-s)^{p(\beta-1)+1-1} s^{(p(\gamma-1)+1) / \alpha-1} d s \\
\quad:=\int_{0}^{1}(1-s)^{n_{2}-1} s^{n_{1}-1} d s,
\end{gathered}
$$

and denote $f(s):=(1-s)^{n_{2}-1} s^{n_{1}-1}$ for $s \in(0,1)$, where $n_{1}=$ $(p(\gamma-1)+1) / \alpha$ and $n_{2}=p(\beta-1)+1$. If $n_{2}=n_{1}$, then $f(s)$ is symmetric about $s=1 / 2$. According to assumption $\left(S_{1}\right)$,

$$
1>n_{1}=\frac{p(\gamma-1)+1}{\alpha}>p(\beta-1)+1=n_{2}>0 ;
$$


that is,

$$
0>n_{1}-1>n_{2}-1>-1 \text {. }
$$

On the other hand, the zero-point of $f^{\prime}(s)$ can be obtained as follows:

$$
s_{0}=\frac{n_{1}-1}{n_{1}+n_{2}-2}<\frac{1}{2} .
$$

Therefore, the function $f(s)$ is decreasing on the interval $\left(0, s_{0}\right]$ while increasing sharply on the interval $\left[s_{0}, 1\right)$. Consequently, for some given sufficiently small $\tau_{k}$, by the properties of the left-rectangle integral formula, we have

$$
\begin{aligned}
& \sum_{k=0}^{n-1}\left(1^{\alpha}-t_{k}^{\alpha}\right)^{n_{2}-1} t_{k}^{n_{1}-1} \tau_{k} \\
& \quad \leq \int_{0}^{1}\left(1-s^{\alpha}\right)^{n_{2}-1} s^{n_{1}-1} d s \\
& \quad=B\left[\frac{p(\gamma-1)+1}{\alpha}, p(\beta-1)+1\right],
\end{aligned}
$$

where $0=t_{0}<t_{1}<\cdots<t_{n}=1$. For the general interval $\left(0, t_{n}\right]$, we can easily obtain the corresponding result (15) by the similar method and omit the details here.

Denote $\widetilde{a}_{n}=\max _{0 \leq k \leq n, k \in \mathbb{N}} a_{k}$ and $\tau=\max _{0 \leq k \leq n-1, k \in \mathbb{N}} \tau_{k}$, where $\tau_{k}$ is the variable time step. Next, we first discuss inequality (7) and obtain the following result.

Theorem 6. Under assumptions $\left(S_{1}\right),\left(S_{2}\right)$, and $\left(S_{3}\right)$, if $x_{n}$ is nonnegative such that (7), then

$$
\begin{aligned}
& x_{n} \leq\left[\Omega ^ { - 1 } \left(\Omega\left(2^{q-1} \widetilde{a}_{n}^{q}\right)+2^{q-1} \tau\left(\frac{t_{n}^{\theta}}{\alpha}\right)^{q / p}\right.\right. \\
&\left.\left.\times\left(B\left[\frac{p(\gamma-1)+1}{\alpha}, p(\beta-1)+1\right]\right)^{q / p} \sum_{k=0}^{n-1} b_{k}^{q}\right)\right]^{1 / q},
\end{aligned}
$$

for $0 \leq n \leq N_{1}$, where $\Omega(u)=\int_{u_{0}}^{u}\left(1 / \omega^{q}\left(s^{1 / q}\right)\right) d s, u \geq u_{0}, \Omega^{-1}$ is the inverse function of $\Omega, \theta=p[\alpha(\beta-1)+\gamma-1]+1$, and $N_{1}$ is the largest integer number such that

$$
\begin{aligned}
\Omega & \left(2^{q-1} \widetilde{a}_{n}^{q}\right)+2^{q-1} \tau\left(\frac{t_{n}^{\theta}}{\alpha}\right)^{q / p} \\
& \times\left(B\left[\frac{p(\gamma-1)+1}{\alpha}, p(\beta-1)+1\right]\right)^{q / p} \\
& \times \sum_{k=0}^{n-1} b_{k}^{q} \in \operatorname{Dom}\left(\Omega^{-1}\right) .
\end{aligned}
$$

Proof. Since $\widetilde{a}_{n}=\max _{0 \leq k \leq n, k \in \mathbb{N}} a_{k}$, according to assumption $\left(S_{2}\right), \tilde{a}_{n}$ is nonnegative and nondecreasing, and $\widetilde{a}_{n} \geq a_{n}$. From (7), we have

$$
x_{n} \leq \tilde{a}_{n}+\sum_{k=0}^{n-1}\left(t_{n}^{\alpha}-t_{k}^{\alpha}\right)^{\beta-1} t_{k}^{\gamma-1} \tau_{k} b_{k} \omega\left(x_{k}\right) .
$$

Due to assumption $\left(S_{1}\right)$, take suitable indices $p, q$ such that $1 / p+1 / q=1$. An application of Lemma 2 yields

$$
\begin{aligned}
x_{n} \leq & \widetilde{a}_{n}+\sum_{k=0}^{n-1}\left(t_{n}^{\alpha}-t_{k}^{\alpha}\right)^{\beta-1} t_{k}^{\gamma-1} \tau_{k}^{1 / p} \tau_{k}^{1 / q} b_{k} \omega\left(x_{k}\right) \\
\leq & \widetilde{a}_{n}+\tau^{1 / q} \sum_{k=0}^{n-1}\left(t_{n}^{\alpha}-t_{k}^{\alpha}\right)^{\beta-1} t_{k}^{\gamma-1} \tau_{k}^{1 / p} b_{k} \omega\left(x_{k}\right) \\
\leq & \widetilde{a}_{n}+\tau^{1 / q}\left[\sum_{k=0}^{n-1}\left(t_{n}^{\alpha}-t_{k}^{\alpha}\right)^{p(\beta-1)} t_{k}^{p(\gamma-1)} \tau_{k}\right]^{1 / p} \\
& \times\left[\sum_{k=0}^{n-1} b_{k}^{q} \omega^{q}\left(x_{k}\right)\right]^{1 / q} .
\end{aligned}
$$

By Lemma 1, it follows from the inequality above that

$$
\begin{aligned}
x_{n}^{q} \leq & 2^{q-1} \widetilde{a}_{n}^{q}+2^{q-1} \tau\left[\sum_{k=0}^{n-1}\left(t_{n}^{\alpha}-t_{k}^{\alpha}\right)^{p(\beta-1)} t_{k}^{p(\gamma-1)} \tau_{k}\right]^{q / p} \\
& \times\left[\sum_{k=0}^{n-1} b_{k}^{q} \omega^{q}\left(x_{k}\right)\right] .
\end{aligned}
$$

Considering

$$
\begin{aligned}
& \sum_{k=0}^{n-1}\left(t_{n}^{\alpha}-t_{k}^{\alpha}\right)^{p(\beta-1)} t_{k}^{p(\gamma-1)} \tau_{k} \\
& \quad \leq \int_{0}^{t_{n}}\left(t_{n}^{\alpha}-s^{\alpha}\right)^{p(\beta-1)} s^{p(\gamma-1)} d s \\
& \quad=\frac{t_{n}^{\theta}}{\alpha} B\left[\frac{p(\gamma-1)+1}{\alpha}, p(\beta-1)+1\right],
\end{aligned}
$$

in which we apply Lemma 5, we have

$$
\begin{aligned}
x_{n}^{q} \leq & 2^{q-1} \widetilde{a}_{n}^{q}+2^{q-1} \tau\left(\frac{t_{n}^{\theta}}{\alpha}\right)^{q / p} \\
& \times\left(B\left[\frac{p(\gamma-1)+1}{\alpha}, p(\beta-1)+1\right]\right)^{q / p} \\
& \times\left[\sum_{k=0}^{n-1} b_{k}^{q} \omega^{q}\left(x_{k}\right)\right] .
\end{aligned}
$$

Let $v_{n}=x_{n}^{q}, A_{n}=2^{q-1} \tilde{a}_{n}^{q}$, and $C_{n}=2^{q-1} \tau\left(t_{n}^{\theta} / \alpha\right)^{q / p}$ $(B[(p(\gamma-1)+1) / \alpha, p(\beta-1)+1])^{q / p}$. Obviously, $A_{n}, C_{n}$ are nondecreasing for $n \in \mathbb{N}$ and $\omega^{q}\left(v_{k}^{1 / q}\right)$ satisfies assumption $\left(S_{3}\right)$. Equation (27) can be rewritten as

$$
v_{n} \leq A_{n}+C_{n}\left(\sum_{k=0}^{n-1} b_{k}^{q} \omega^{q}\left(v_{k}^{1 / q}\right)\right),
$$

which is similar to inequality (12). Using Lemma 4 to (28), we have

$$
v_{n} \leq\left[\Omega^{-1}\left(\Omega\left(A_{n}\right)+C_{n} \sum_{k=0}^{n-1} b_{k}^{q}\right)\right],
$$


for $0 \leq n \leq N_{1}$, where $\Omega(u)=\int_{u_{0}}^{u}\left(1 / \omega^{q}\left(s^{1 / q}\right)\right) d s, u \geq u_{0}$, $\Omega^{-1}$ is the inverse function of $\Omega$, and $N_{1}$ is the largest integer number such that

$$
\Omega\left(A_{n}\right)+C_{n} \sum_{k=0}^{n-1} b_{k}^{q} \in \operatorname{Dom}\left(\Omega^{-1}\right) .
$$

Therefore, by $x_{n}=v_{n}^{1 / q},(21)$ holds for $0 \leq n \leq N_{1}$.

Remark 7. When $\alpha=1$ and $\gamma=1$, the inequality was discussed by Medved [12] which is the special case of our result. Moreover, his result holds under the assumption " $\omega(u)$ satisfies the condition $(q)$;" that is, " $e^{-q t}[\omega(u)]^{q} \leq$ $R(t) \omega\left(e^{-q t} u^{q}\right)$, where $R(t)$ is a continuous, nonnegative function." In our result, the condition $(q)$ is eliminated.

Corollary 8. Under assumptions $\left(S_{1}\right)$ and $\left(S_{2}\right)$, let $\nu>0, \mu>$ $0(\nu>\mu)$. If $x_{n}$ is nonnegative such that

$$
x_{n}^{\nu} \leq a_{n}+\sum_{k=0}^{n-1}\left(t_{n}^{\alpha}-t_{k}^{\alpha}\right)^{\beta-1} t_{k}^{\gamma-1} \tau_{k} b_{k} x_{k}^{\mu},
$$

then

$$
\begin{aligned}
x_{n} \leq & {\left[2^{q-1} \widetilde{a}_{n}^{(\nu-\mu) / \nu}+\frac{\nu-\mu}{\nu} 2^{q-1} \tau\left(\frac{t_{n}^{\theta}}{\alpha}\right)^{q / p}\right.} \\
& \times\left(B\left[\frac{p(\gamma-1)+1}{\alpha}, p(\beta-1)+1\right]\right)^{q / p_{n-1}} \sum_{k=0}^{q} b_{k}^{1 /(\nu-\mu) q}
\end{aligned}
$$

for $n \geq 0$.

Proof. Let $y_{n}=x_{n}^{\nu}$; then $x_{n}=y_{n}^{1 / v}$ or $x_{n}^{\mu}=y_{n}^{\mu / v}$. From (31) we have

$$
y_{n} \leq a_{n}+\sum_{k=0}^{n-1}\left(t_{n}^{\alpha}-t_{k}^{\alpha}\right)^{\beta-1} t_{k}^{\gamma-1} \tau_{k} b_{k} y_{k}^{\mu / \nu} .
$$

Denote $\omega\left(y_{k}\right)=y_{k}^{\mu / \nu}$. Clearly, $\omega$ satisfies assumption $\left(S_{3}\right)$. With the definition of $\Omega$ in Theorem 6 , letting $u_{0}=0$, we have

$$
\begin{gathered}
\Omega(u)=\int_{0}^{u} \frac{d s}{s^{\mu / \nu}}=\frac{v}{v-\mu} u^{(\nu-\mu) / \nu} \\
\Omega^{-1}(u)=\left(\frac{\nu-\mu}{\nu} u\right)^{\nu /(\nu-\mu)}, \quad \operatorname{Dom}\left(\Omega^{-1}\right)=[0, \infty) .
\end{gathered}
$$

Substituting (34) and (35) into (29), we get

$$
\begin{aligned}
y_{n} \leq & {\left[2^{q-1} \widetilde{a}_{n}^{(\nu-\mu) / \nu}+\frac{\nu-\mu}{\nu} 2^{q-1} \tau\left(\frac{t_{n}^{\theta}}{\alpha}\right)^{q / p}\right.} \\
& \left.\times\left(B\left[\frac{p(\gamma-1)+1}{\alpha}, p(\beta-1)+1\right]\right)^{q / p} \sum_{k=0}^{n-1} b_{k}^{q}\right]^{\nu /(\nu-\mu) q} .
\end{aligned}
$$

In view of $x_{n}=y_{n}^{1 / v}$, we can obtain (32).
Remark 9. In [15], Yang et al. investigated inequality (6). Clearly, let $\alpha=1$ and $\gamma=1$ in (31), and we can get the same formula.

Remark 10. Let $\nu=2$ and $\mu=1$; we can get the interesting Henry's version of the Ou-Iang-Pachpatte type difference inequality [26]. Thus, our results are a more general discrete analogue for such inequality.

Remark 11. Ma and Pečarić discussed the continuous case of (2.15) in [27] and here we present the discrete version of their result. Furthermore, the result in [27] is established for the cases when the ordered parameter group $[\alpha, \beta, \gamma]$ obeys distribution I or II (for details, see [27]) which makes the application of inequality more inconvenient. Clearly, our result is based on the concise assumption to overcome this weakness.

Corollary 12. Under assumptions $\left(S_{1}\right)$ and $\left(S_{2}\right)$, if $x_{n}$ is nonnegative such that

$$
x_{n} \leq a_{n}+\sum_{k=0}^{n-1}\left(t_{n}^{\alpha}-t_{k}^{\alpha}\right)^{\beta-1} t_{k}^{\gamma-1} \tau_{k} b_{k} x_{k}
$$

then

$$
\begin{aligned}
x_{n} \leq 2^{(q-1) / q} & \widetilde{a}_{n} \\
\times \exp & \left(\frac{2^{q-1}}{q} \tau\left(\frac{t_{n}^{\theta}}{\alpha}\right)^{q / p}\right. \\
& \left.\times\left(B\left[\frac{p(\gamma-1)+1}{\alpha}, p(\beta-1)+1\right]\right)^{q / p} \sum_{k=0}^{n-1} b_{k}^{q}\right),
\end{aligned}
$$

for $n \geq 0$.

Proof. In (7), $\omega(u)=u$ also satisfies assumption $\left(S_{3}\right)$. Thus, we have

$$
\begin{gathered}
\Omega(u)=\int_{u_{0}}^{u} \frac{d s}{s}=\ln \frac{u}{u_{0}}, \\
\Omega^{-1}(u)=u_{0} \exp (u), \\
\operatorname{Dom}\left(\Omega^{-1}\right)=[0, \infty) .
\end{gathered}
$$

Similar to the computation in Corollary 8, estimate (38) holds.

Now, we discuss inequality (8)

$$
\begin{aligned}
x_{n} \leq & a_{n}+\sum_{k=0}^{n-1}\left(t_{n}^{\sigma}-t_{k}^{\sigma}\right)^{\mu-1} t_{k}^{\lambda-1} \tau_{k} g_{k} x_{k} \\
& +\sum_{k=0}^{n-1}\left(t_{n}^{\alpha}-t_{k}^{\alpha}\right)^{\beta-1} t_{k}^{\gamma-1} \tau_{k} b_{k} \omega\left(x_{k}\right) .
\end{aligned}
$$


Since there are two different parameter groups $[\sigma, \mu, \lambda]$ and $[\alpha, \beta, \gamma]$, assumption $\left(S_{1}\right)$ is revised as follows:

$\left(S_{4}\right) \sigma \in(0,1], \mu \in(0,1), 1>(p(\lambda-1)+1) / \sigma \geq p(\mu-1)+$ $1>0$ and $\alpha \in(0,1], \beta \in(0,1), 1>(p(\gamma-1)+1) / \alpha \geq$ $p(\beta-1)+1>0$ such that $1 / p+\sigma(\mu-1)+\lambda-1 \geq 0$ and $1 / p+\alpha(\beta-1)+\gamma-1 \geq 0(p>1)$.

Theorem 13. Under assumptions $\left(S_{3}\right)$ and $\left(S_{4}\right)$, suppose that $g_{k}, b_{k}$ are nonnegative for $n \in \mathbb{N}$. If $x_{n}$ is nonnegative such that (8), then

$$
\begin{aligned}
x_{n} \leq\left[\Omega^{-1}(\right. & \Omega\left(2^{q-1} \widetilde{a}_{n}^{q} Q_{n}^{q}\right)+2^{q-1} Q_{n}^{q} \tau\left(\frac{t_{n}^{\theta_{2}}}{\alpha}\right)^{q / p} \\
& \times\left(B\left[\frac{p(\gamma-1)+1}{\alpha}, p(\beta-1)+1\right]\right)^{q / p} \\
& \left.\left.\times \sum_{k=0}^{n-1} b_{k}^{q}\right)\right]^{1 / q},
\end{aligned}
$$

for $0 \leq n \leq N_{2}$, where $\Omega$ and $\Omega^{-1}$ are defined as in Theorem 6 ,

$$
Q_{n}=2^{(q-1) / q}
$$

$$
\begin{aligned}
\times \exp \left(\frac{2^{q-1}}{q} \tau\left(\frac{t_{n}^{\theta_{1}}}{\sigma}\right)^{q / p}\right. \\
\left.\times\left(B\left[\frac{p(\lambda-1)+1}{\sigma}, p(\mu-1)+1\right]\right)^{q / p} \sum_{k=0}^{n-1} g_{k}^{q}\right), \\
\theta_{1}=p[\sigma(\mu-1)+\lambda-1]+1, \\
\theta_{2}=p[\alpha(\beta-1)+\gamma-1]+1,
\end{aligned}
$$

and $N_{2}$ is the largest integer number such that

$$
\begin{aligned}
\Omega & \left(2^{q-1} \widetilde{a}_{n}^{q} Q_{n}^{q}\right)+2^{q-1} Q_{n}^{q} \tau\left(\frac{t_{n}^{\theta_{2}}}{\alpha}\right)^{q / p} \\
& \times\left(B\left[\frac{p(\gamma-1)+1}{\alpha}, p(\beta-1)+1\right]\right)^{q / p} \\
& \times \sum_{k=0}^{n-1} b_{k}^{q} \in \operatorname{Dom}\left(\Omega^{-1}\right) .
\end{aligned}
$$

Proof. By the definition of $\tilde{a}_{n}$, we have

$$
\begin{aligned}
x_{n} \leq & \tilde{a}_{n}+\sum_{k=0}^{n-1}\left(t_{n}^{\sigma}-t_{k}^{\sigma}\right)^{\mu-1} t_{k}^{\lambda-1} \tau_{k} g_{k} x_{k} \\
& +\sum_{k=0}^{n-1}\left(t_{n}^{\alpha}-t_{k}^{\alpha}\right)^{\beta-1} t_{k}^{\gamma-1} \tau_{k} b_{k} \omega\left(x_{k}\right) .
\end{aligned}
$$

Let

$$
P_{n}=\tilde{a}_{n}+\sum_{k=0}^{n-1}\left(t_{n}^{\alpha}-t_{k}^{\alpha}\right)^{\beta-1} t_{k}^{\gamma-1} \tau_{k} b_{k} \omega\left(x_{k}\right),
$$

which yields directly

$$
x_{n} \leq P_{n}+\sum_{k=0}^{n-1}\left(t_{n}^{\sigma}-t_{k}^{\sigma}\right)^{\mu-1} t_{k}^{\lambda-1} \tau_{k} g_{k} x_{k} .
$$

Using Corollary 12, from the inequality above, we get

$$
\begin{aligned}
x_{n} \leq 2^{(q-1) / q} & P_{n} \\
\times \exp & \left(\frac{2^{q-1}}{q} \tau\left(\frac{t_{n}^{\theta_{1}}}{\sigma}\right)^{q / p}\right. \\
& \left.\times\left(B\left[\frac{p(\lambda-1)+1}{\sigma}, p(\mu-1)+1\right]\right)^{q / p} \sum_{k=0}^{n-1} g_{k}^{q}\right),
\end{aligned}
$$

where $\theta_{1}=p[\sigma(\mu-1)+\lambda-1]+1$. Letting

$$
\begin{aligned}
Q_{n}=2^{(q-1) / q} \exp \left(\frac{2^{q-1}}{q} \tau\left(\frac{t_{n}^{\theta_{1}}}{\sigma}\right)^{q / p}\right. \\
\quad \times\left(B\left[\frac{p(\lambda-1)+1}{\sigma}, p(\mu-1)+1\right]\right)^{q / p} \\
\left.\times \sum_{k=0}^{n-1} g_{k}^{q}\right),
\end{aligned}
$$

from (47), we get

$$
u_{n} \leq P_{n} Q_{n}
$$

$$
\leq \tilde{a}_{n} Q_{n}+Q_{n} \sum_{k=0}^{n-1}\left(t_{n}^{\alpha}-t_{k}^{\alpha}\right)^{\beta-1} t_{k}^{\gamma-1} \tau_{k} b_{k} \omega\left(x_{k}\right) .
$$

Clearly, inequality (49) is similar to (7). According to Theorem 6, we obtain

$$
\begin{aligned}
& x_{n} \leq\left[\Omega ^ { - 1 } \left(\Omega\left(2^{q-1} \widetilde{a}_{n}^{q} Q_{n}^{q}\right)+2^{q-1} Q_{n}^{q} \tau\left(\frac{t_{n}^{\theta_{2}}}{\alpha}\right)^{q / p}\right.\right. \\
& \times\left(B\left[\frac{p(\gamma-1)+1}{\alpha}, p(\beta-1)+1\right]\right)^{q / p} \\
&\left.\left.\times \sum_{k=0}^{n-1} b_{k}^{q}\right)\right]^{1 / q},
\end{aligned}
$$


Remark 14. Our result for inequality (8) is also the discrete analogue of inequality (5). In fact, with the different choice of the parameter groups $[\alpha, \beta, \gamma]$ and $[\sigma, \mu, \lambda]$ in $[14]$, the complicate results must be presented by four cases, respectively. Apparently, compared to their results, our result is quite simple.

\section{Applications}

In this section, we apply our results to discuss the upper bound and the uniqueness of solutions of a Volterra type difference equation with certain weakly singular kernels.

Example 15. Consider the following inequality:

$$
\begin{aligned}
x_{n} \leq & \frac{1}{2}+\sum_{k=0}^{n-1}\left(t_{n}-t_{k}\right)^{-1 / 3} t_{k}^{-1 / 4} \tau_{k} x_{k} \\
& +\sum_{k=0}^{n-1}\left(t_{n}-t_{k}\right)^{-1 / 3} t_{k}^{-1 / 3} \tau_{k} \sqrt{x_{k}} .
\end{aligned}
$$

Obviously, (51) is the special case of inequality (8), and we get

$$
\begin{gathered}
a_{n}=\frac{1}{2}, \quad \sigma=1, \quad \mu=\frac{2}{3}, \quad \lambda=\frac{3}{4}, \\
\alpha=1, \quad \beta=\frac{2}{3}, \quad \gamma=\frac{2}{3}, \\
g_{k}=1, \quad b_{k}=1 .
\end{gathered}
$$

Next, we discuss the choice of parameter $p$. By assumption $\left(S_{4}\right)$, from the conditions $1>(p(\lambda-1)+1) / \sigma \geq p(\mu-1)+1>0$ and $1 / p+\sigma(\mu-1)+\lambda-1 \geq 0$, we have $1<p<12 / 7$. From the conditions $1>(p(\gamma-1)+1) / \alpha \geq p(\beta-1)+1>0$ and $1 / p+\alpha(\beta-1)+\gamma-1 \geq 0(p>1)$, we have $1<p<3 / 2$. Thus, we can take $p=4 / 3$; then $q=4, q / p=3$. According to Theorem 13, we obtain

$$
\begin{gathered}
\tilde{a}_{n}=\frac{1}{2}, \quad \theta_{1}=\frac{2}{9}, \quad \theta_{2}=\frac{1}{9}, \\
B\left[\frac{p(\lambda-1)+1}{\sigma}, p(\mu-1)+1\right]=B\left[\frac{2}{3}, \frac{5}{9}\right], \\
B\left[\frac{p(\gamma-1)+1}{\alpha}, p(\beta-1)+1\right]=B\left[\frac{5}{9}, \frac{5}{9}\right], \\
Q_{n}=2^{3 / 4} \exp \left(2 \tau\left(t_{n}^{2 / 9}\right)^{3}\left(B\left[\frac{2}{3}, \frac{5}{9}\right]\right)^{3} n\right), \\
\Omega(u)=\int_{0}^{u} \frac{d s}{\sqrt{s}}=2 \sqrt{u}, \quad \Omega^{-1}(u)=\frac{u^{2}}{4}, \\
2^{q-1} \widetilde{a}_{n}^{q} Q_{n}^{q}=4 \exp \left(8 \tau t_{n}^{1 / 5} B^{3}\left[\frac{2}{3}, \frac{5}{9}\right] n\right) .
\end{gathered}
$$

Substituting the results above into (41), we can get the upper bound of $x_{n}$ and omit the details for its complicated formula.
Example 16. Consider the linear weakly singular difference equation

$$
\begin{aligned}
& x_{n}=a_{n}+\sum_{k=0}^{n-1}\left(t_{n}^{\alpha}-t_{k}^{\alpha}\right)^{\beta-1} t_{k}^{\gamma-1} \tau_{k} b_{k} x_{k}, \\
& y_{n}=c_{n}+\sum_{k=0}^{n-1}\left(t_{n}^{\alpha}-t_{k}^{\alpha}\right)^{\beta-1} t_{k}^{\gamma-1} \tau_{k} b_{k} y_{k},
\end{aligned}
$$

where $\left|a_{n}-c_{n}\right|<\epsilon, \epsilon$ is an arbitrary positive number, and the parameter group $[\alpha, \beta, \gamma]$ satisfies assumption $\left(S_{1}\right)$. From (54) and (55), we get

$$
\left|x_{n}-y_{n}\right| \leq\left|a_{n}-c_{n}\right|+\sum_{k=0}^{n-1}\left(t_{n}^{\alpha}-t_{k}^{\alpha}\right)^{\beta-1} t_{k}^{\gamma-1} \tau_{k} b_{k}\left|x_{k}-y_{k}\right|,
$$

which is the form of inequality (37). Applying Corollary 12, we have

$$
\begin{aligned}
\left|x_{n}-y_{n}\right| \leq 2^{(q-1) / q} & \epsilon \\
& \times \exp \left(\frac{2^{q-1}}{q} \tau\left(\frac{t_{n}^{\theta}}{\alpha}\right)^{q / p}\right. \\
& \times\left(B\left[\frac{p(\gamma-1)+1}{\alpha}, p(\beta-1)+1\right]\right)^{q / p} \\
& \left.\times \sum_{k=0}^{n-1} b_{k}^{q}\right)
\end{aligned}
$$

for $n \in \mathbb{N}$. If $a_{n}=c_{n}$, let $\epsilon \rightarrow 0$ and we obtain the uniqueness of the solution of (54).

\section{Conflict of Interests}

The authors declare that there is no conflict of interests regarding the publication of this paper.

\section{Acknowledgments}

The authors are very grateful to the referees for their helpful comments and valuable suggestions. This work is supported by the Doctoral Program Research Funds of Southwest University of Science and Technology (no. 11zx7129) and the Fundamental Research Funds for the Central Universities (no. skqy201324).

\section{References}

[1] R. P. Agarwal, S. Deng, and W. Zhang, "Generalization of a retarded Gronwall-like inequality and its applications," Applied Mathematics and Computation, vol. 165, no. 3, pp. 599-612, 2005.

[2] S. Deng, "Nonlinear discrete inequalities with two variables and their applications," Applied Mathematics and Computation, vol. 217, no. 5, pp. 2217-2225, 2010. 
[3] A. A. Martyniuk, V. Lakshamikantham, and S. Leela, Motion Stability: The Method of Integral Inequalities, Naukova Dumka, Kiev, Russia, 1977.

[4] B. G. Pachpatte, "On some fundamental integral inequalities and their discrete analogues," Journal of Inequalities in Pure and Applied Mathematics, vol. 2, no. 2, article 15, 2001.

[5] S. Deng, Y. Wu, and X. Li, "Nonlinear delay discrete inequalities and their applications to Volterra type difference equations," Advances in Difference Equations, vol. 2010, Article ID 795145, 14 pages, 2010.

[6] P. R. Beesack, "More generalised discrete Gronwall inequalities," Zeitschrift für Angewandte Mathematik und Mechanik, vol. 65, no. 12, pp. 583-595, 1985.

[7] J. Dixon and S. McKee, "Weakly singular discrete Gronwall inequalities," Zeitschrift für Angewandte Mathematik und Mechanik, vol. 66, no. 11, pp. 535-544, 1986.

[8] J. P. Dauer and N. I. Mahmudov, "Integral inequalities and mild solutions of semilinear neutral evolution equations," Journal of Mathematical Analysis and Applications, vol. 300, no. 1, pp. 189202, 2004.

[9] D. Henry, Geometric Theory of Semilinear Parabolic Equations, vol. 840 of Lecture Notes in Mathematics, Springer, New York, NY, USA, 1981.

[10] J. Januszewski, "On Volterra integral equations with weakly singular kernels in Banach spaces," Demonstratio Mathematica, vol. 26, no. 1, pp. 131-136, 1993.

[11] M. Medved', "A new approach to an analysis of Henry type integral inequalities and their Bihari type versions," Journal of Mathematical Analysis and Applications, vol. 214, no. 2, pp. 349366, 1997.

[12] M. Medved, "Nonlinear singular difference inequalities suitable for discretizations of parabolic equations," Demonstratio Mathematica, vol. 33, no. 3, pp. 517-525, 2000.

[13] M. Medved, "On singular versions of Bihari and WendroffPachpatte type integral inequalities and their application," Tatra Mountains Mathematical Publications, vol. 38, pp. 163-174, 2007.

[14] Q. H. Ma and E. H. Yang, "Estimates on solutions of some weakly singular Volterra integral inequalities," Acta Mathematicae Applicatae Sinica, vol. 25, no. 3, pp. 505-515, 2002.

[15] E. H. Yang, Q. H. Ma, and M. C. Tan, "Discrete analogues of a new class of nonlinear Volterra singular integral inequalitie," Journal of Jinan University, vol. 28, no. 1, pp. 1-6, 2007.

[16] W. S. Cheung, Q. H. Ma, and S. Tseng, "Some new nonlinear weakly singular integral inequalities of wendroff type with applications," Journal of Inequalities and Applications, vol. 2008, Article ID 909156, 12 pages, 2008.

[17] S. Deng and C. Prather, "Generalization of an impulsive nonlinear singular Gronwall-Bihari inequality with delay," Journal of Inequalities in Pure and Applied Mathematics, vol. 9, no. 2, article 34, 2008.

[18] K. M. Furati and N. Tatar, "Power-type estimates for a nonlinear fractional differential equation," Nonlinear Analysis: Theory, Methods \& Applications, vol. 62, no. 6, pp. 1025-1036, 2005.

[19] F. Lakhal, "A new nonlinear integral inequality of Wendroff type with continuous and weakly singular kernel and its application," Journal of Mathematical Inequalities, vol. 6, no. 3, pp. 367-379, 2012.

[20] S. Mazouzi and N.-E. Tatar, "New bounds for solutions of a singular integro-differential inequality," Mathematical Inequalities \& Applications, vol. 13, no. 2, pp. 427-435, 2010.
[21] S. Szufla, "On the Volterra integral equation with weakly singular kernel," Mathematica Bohemica, vol. 131, no. 3, pp. 225231, 2006.

[22] S. Szufla, "On the Hammerstein integral equation with weakly singular kernel," Funkcialaj Ekvacioj, vol. 34, no. 2, pp. 279-285, 1991.

[23] N.-E. Tatar, "On an integral inequality with a kernel singular in time and space," Journal of Inequalities in Pure and Applied Mathematics, vol. 4, no. 4, article 82, 9 pages, 2003.

[24] H. Wang and K. Zheng, "Some nonlinear weakly singular integral inequalities with two variables and applications," Journal of Inequalities and Applications, vol. 2010, Article ID 345701, 2010.

[25] K. Zheng, "Bounds on some new weakly singular Wendrofftype integral inequalities and applications," Journal of Inequalities and Applications, vol. 2013, article 159, 2013.

[26] B. G. Pachpatte, "On some new inequalities related to certain inequalities in the theory of differential equations," Journal of Mathematical Analysis and Applications, vol. 189, no. 1, pp. 128144, 1995.

[27] Q. H. Ma and J. Pečarić, "Some new explicit bounds for weakly singular integral inequalities with applications to fractional differential and integral equations," Journal of Mathematical Analysis and Applications, vol. 341, no. 2, pp. 894-905, 2008. 


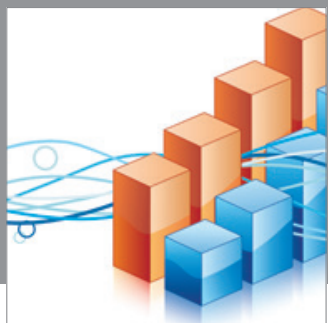

Advances in

Operations Research

mansans

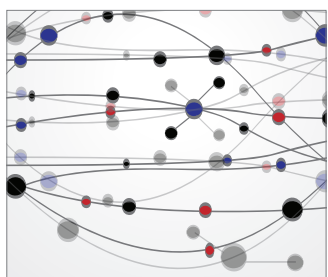

The Scientific World Journal
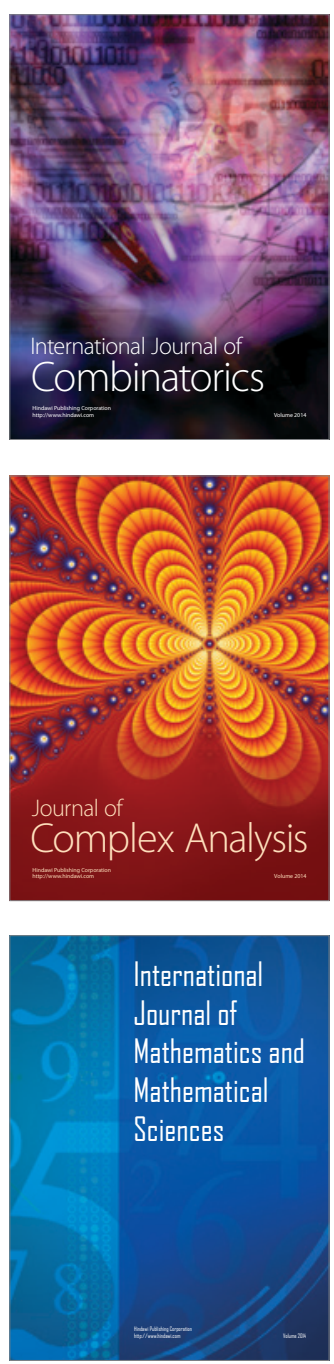
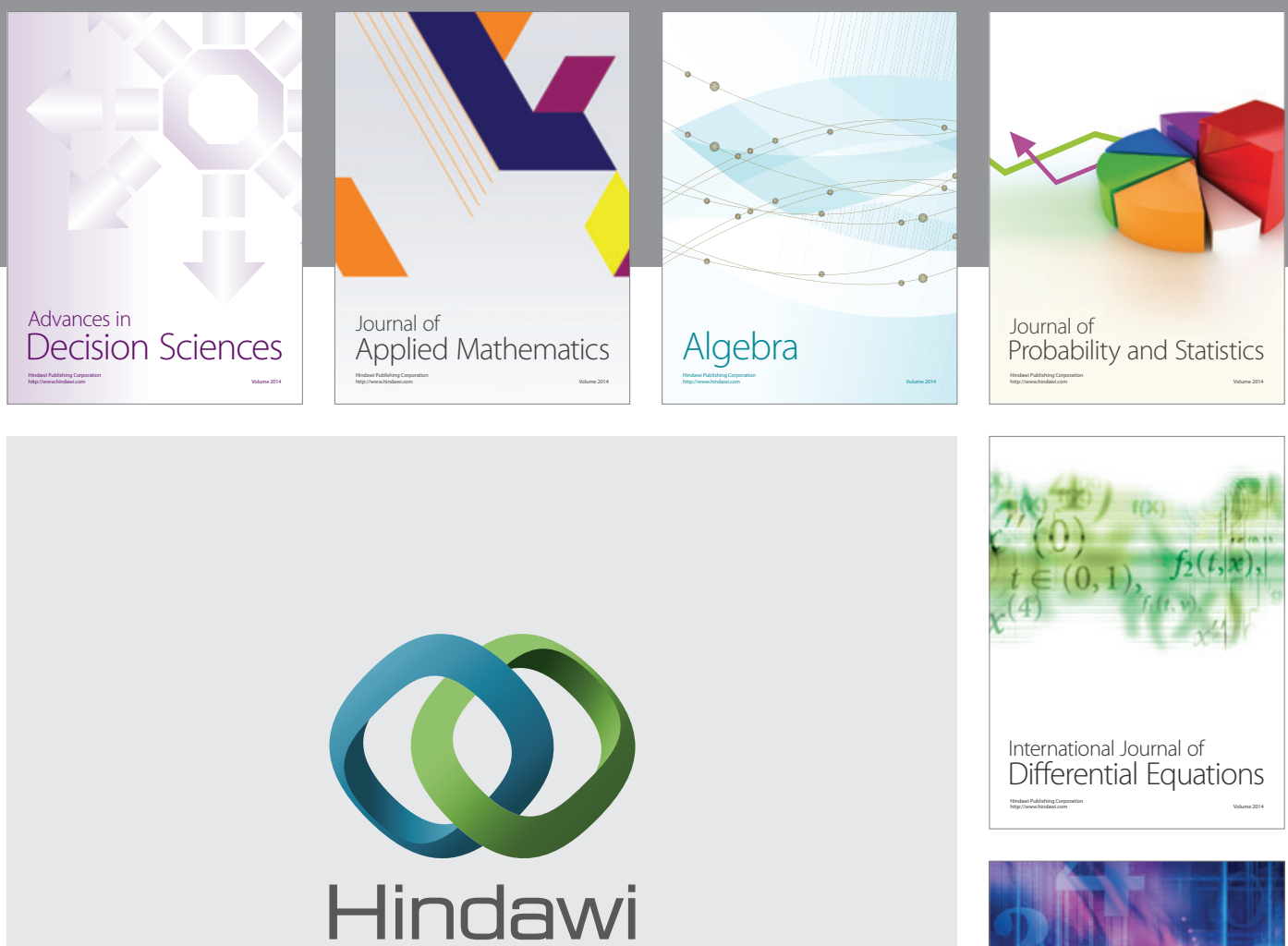

Submit your manuscripts at http://www.hindawi.com
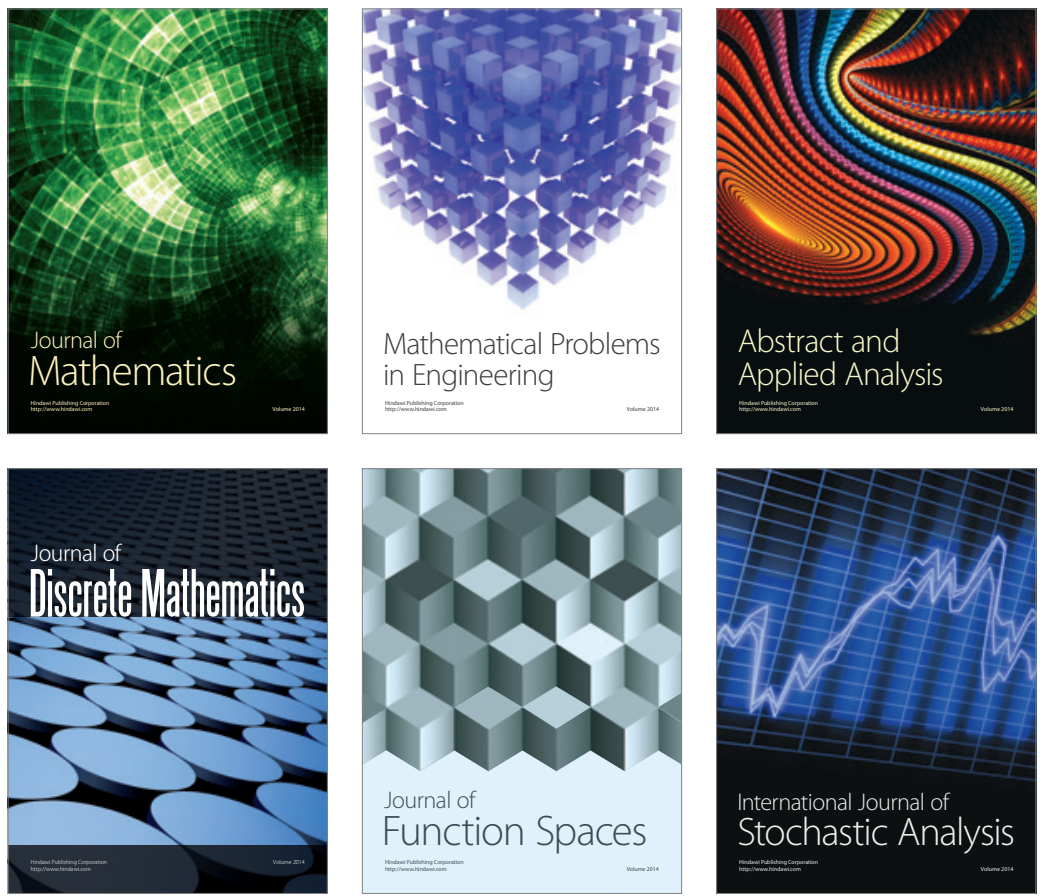

Journal of

Function Spaces

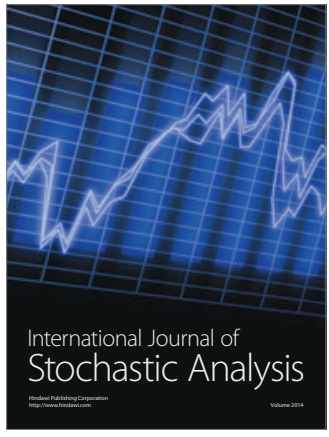

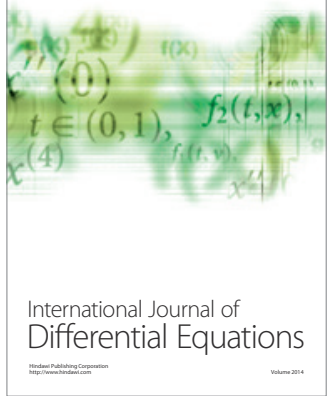
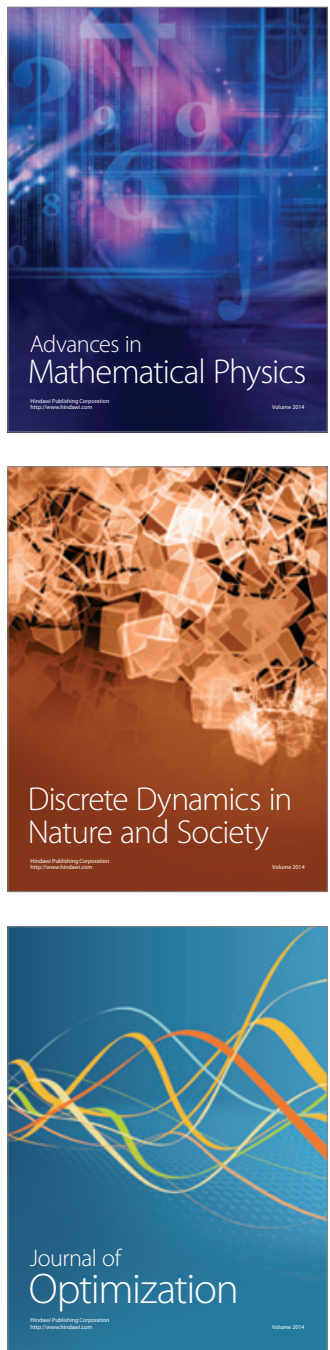